\title{
Proresolving receptor tames inflammation in atherosclerosis
}

\author{
Hebe Agustina Mena and Matthew Spite \\ Center for Experimental Therapeutics and Reperfusion Injury, Department of Anesthesiology, Perioperative and Pain Medicine, Brigham and Women's Hospital and Harvard Medical School, \\ Boston, Massachusetts, USA.
}

\begin{abstract}
Nonresolving inflammation contributes to the progression of atherosclerosis, a chronic disease characterized by the accumulation of lipid-rich arterial plaques infiltrated with immune cells. In this issue of the JCI, Arnardottir and Thul et al. report that GPR32, a receptor for proresolving lipid mediators including resolvin D1, was decreased in human atherosclerotic lesions and that overexpression of this human receptor in mice reduced lesion area and necrosis of atherosclerotic plaques. Mechanistically, GPR32 signaling blunted the production of proinflammatory cytokines, enhanced macrophage phagocytosis, and reduced leukocyte accumulation. These results suggest that therapeutic targeting of GPR32 could be an approach to resolving chronic inflammation in atherosclerosis.
\end{abstract}

\section{Proresolving receptors actively promote the resolution of inflammation}

The acute inflammatory response protects the host, yet is actively resolved to prevent tissue injury and facilitate repair/regeneration. During resolution, polymorphonuclear neutrophil (PMN) recruitment is dampened and phagocyte-mediated removal of apoptotic (i.e., efferocytosis) and necrotic cells ensues. Macrophages are key expert phagocytes in resolution that are temporally reprogrammed to facilitate tissue repair, which associates with decreased proinflammatory mediators and a reciprocal increase in antiinflammatory mediators and growth factors. These key functions in resolution are regulated in part by a superfamily of lipid autacoids termed specialized proresolving mediators (SPMs), the discovery of which helped to define resolution as an active biological process (1).

The SPMs comprise distinct chemical mediators enzymatically biosynthesized from polyunsaturated fatty acids. These mediators include the E-series and D-series resolvins, derived from omega-3 fatty acids eicosapentaenoic acid and docosahexaenoic acid, respectively, and lipoxins, derived from omega- 6 fatty acid arachidonic acid. The biological actions of SPMs are largely mediated by GPCRs expressed in leukocytes and vascular cells (2). For example, resolvin D1 (RvD1; 7S,8R,17S-trihydroxy-4Z,9E,11E,13Z,15E,19Z-docosahexaenoic acid) and its aspirin-triggered (AT) $17 R$ epimer are ligands for two distinct human GPCRs termed ALX/FPR2 and GPR32 (also known as DRV1; ref. 3). Authentic RvD1 binds and activates these receptors with high potency $\left(\mathrm{EC}_{50}\right.$ is $\sim 1-10 \mathrm{pM}$ ) and initiates downstream signaling, leading to regulation of proinflammatory cytokines and microRNAs, reduced PMN-endothelial cell (PMN-EC) interactions, and enhanced macrophage phagocytosis (3-5). In vivo, the proresolving and tissue-reparative actions of RvD1 are lost in mice with $A l x /$ Fpr2 deficien-

\section{Related Article: https://doi.org/10.1172/JCl142883}

Conflict of interest: MS is an inventor on an awarded patent assigned to Brigham and Women's Hospital titled "Antiinflammatory particles" (US patent 10,154,977).

Copyright: (c) 2021, American Society for Clinical Investigation.

Reference information: / Clin Invest. 2021;131(24):e155240. https://doi.org/10.1172/JCI155240.

cy, while overexpression of human $A L X /$ FPR2 enhances resolution (2, 4-6). Nonetheless, the biological roles of ALX/FPR2 in vivo are time and context dependent, given that both proinflammatory (e.g., serum amyloid A) and other proresolving mediators (e.g., lipoxin $\mathrm{A}_{4}$ ) are ligands. In contrast, there is no murine homolog of GPR32, and thus its in vivo role in resolution has been uncharacterized to date. In this issue of the JCI, Arnardottir and Thul et al. address this gap by reporting on their generation of mice with Tg overexpression of human GPR32 on an Apoe- and Alx/Fpr2-deficient background and demonstrate a proresolving role of GPR32 in acute inflammation as well as chronic unresolved inflammation associated with atherosclerosis (7).

\section{Nonresolving inflammation} plays a role in atherosclerosis progression

Atherosclerosis is a chronic vascular disease in which subendothelial retention of modified LDLs initiates maladaptive inflammation. Atherosclerotic plaques are characterized by the accumulation of innate and adaptive immune cells, macrophage and vascular smooth muscle cell-derived (VSMC-derived) foam cells, and production of proinflammatory cytokines, chemokines, and lipid mediators (8). The formation of a necrotic core and thin fibrous cap are characteristics of advanced lesions prone to rupture, which can initiate thrombosis and lead to myocardial infarction and stroke. Defective macrophage efferocytosis is a causal factor in the development of advanced plaques, suggestive of impaired resolution mechanisms (8). Indeed, the ratio of SPMs (e.g., resolvins) to proinflammatory lipid mediators (e.g., leukotrienes) is decreased in vulnerable human plaques, which is also reflected in the saliva of individuals with cardiovascular disease (9-11). The ratio of SPMs to proinflammatory lipid mediators is positively associated with angiographically defined atherosclerosis regression in patients with stable coronary 


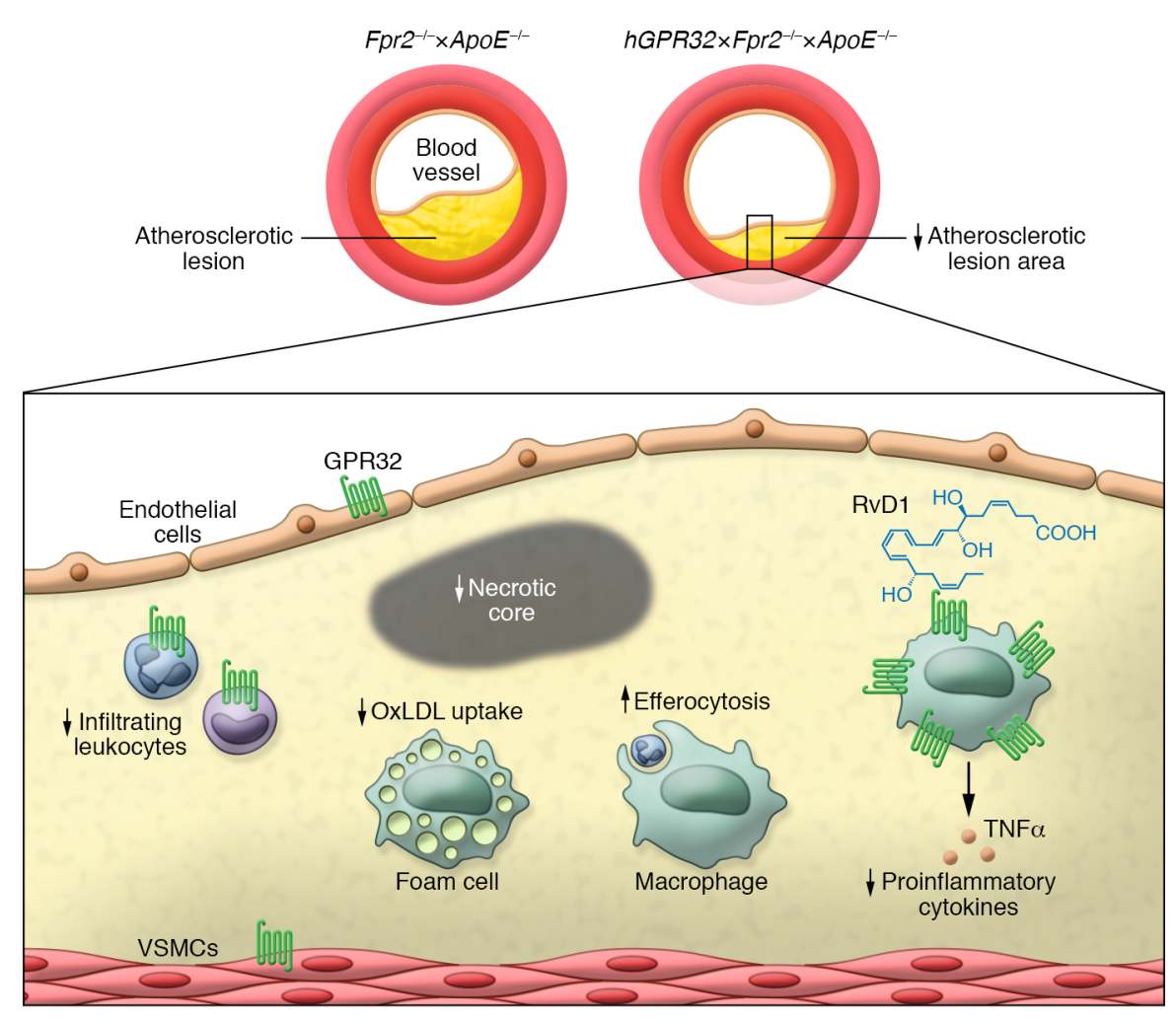

artery disease (12). Mechanistic studies in animal models of atherosclerosis collectively demonstrate that increasing SPMs resolves plaque inflammation, improves efferocytosis, decreases necrotic cores, and increases markers of lesion stability (i.e., fibrous cap thickening; refs. 8, 9,11,13). SPMs also diminish the extent of injury following vascular and endovascular surgical procedures, in part by targeting proliferative VSMCs and inflammation (14). Arnardottir and Thul et al. add to this growing body of literature and establish the proresolving and atheroprotective roles of GPR32 (7).

\section{Proresolving actions of GPR32 in atherosclerosis}

Proresolving receptors, such as ALX/ FPR2 and ERV1/ChemR23 (a receptor for resolvin E1; RvE1), are expressed in both immune cells and vascular cells of human atherosclerotic plaques. Further, ALX/ FPR2 levels are increased in atherosclerosis $(8,15)$. In contrast, Arnardottir and Thul et al. observed that human atherosclerotic plaques show decreased GPR32 mRNA levels compared with control arteries and showed that GPR32 mRNA levels positively correlated with expression of genes encoding M2 macrophage marker $A R G 1$, Treg marker FOXP3, and Th2 cyto- kine $I L 4$ as well as M1 macrophage marker NOS2. At the protein level, GPR32 was expressed in $\mathrm{ARG}^{+}$macrophages and to a lower extent in T cells, VSMCs, and ECs (Figure 1). The authors hypothesized that increasing its expression may have atheroprotective roles in vivo, acting by resolving plaque inflammation. To this end, they generated a mouse strain in which human GPR32 (hGPR32) was expressed in atherosclerosis-prone Apoe-deficient mice that also lacked murine Alx/Fpr2 (7). This approach was needed because SPMs that activate GPR32 also target ALX/FPR2 (2). After high-fat diet feeding, hGPR32 $\mathrm{Tg}$ mice had smaller atherosclerotic lesions as compared with nontransgenic littermates. Features of advanced atherosclerosis, such as necrotic cores, were reduced in hGPR32 $\mathrm{Tg}$ mice, and this reduction coincided with reduction in leukocytes and the proinflammatory cytokine TNF- $\alpha$. These atheroprotective effects occurred independently of changes in circulating immune cells.

The results of the study by Arnardottir and Thul et al. are congruent with prior studies demonstrating that loss of Erv1/ ChemR23 in atherosclerosis-prone mice worsens lesion development and that increasing RvE1 reduces atherosclerosis in both mice and rabbits $(16,17)$. Moreover,
Figure 1. The role of human GPR32 in atherosclerosis. Tg overexpression of $h G P R 32$ on an Apoe- and Fpr2-deficient background reduces atherosclerotic lesion area and necrotic cores as compared with that of non-Tg $\mathrm{Fpr}^{-/-} \times \mathrm{A}^{-}$

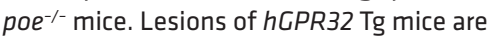
characterized by a lower number of monocytes and neutrophils, reduced macrophages, and decreased levels of the proinflammatory cytokine TNF- $\alpha$. Of note, overexpression of hCPR32 also increases macrophage efferocytosis in peritonitis, and RvD1 enhances macrophage phagocytosis and lowers the uptake of oxidized LDL by macrophages in vitro in an hGPR32-dependent manner. These results suggests that GPR32 has multiple proresolving and atheroprotective roles. Similarly, RvD1 binds GPR32, enhances phagocytosis, and lowers the uptake of oxidized LDL.

RvD1 treatment in $\mathrm{Ldlr}^{-/-}$mice diminishes necrotic cores, which is similar to what is observed in hGPR32 Tg mice and indicative of enhanced efferocytosis $(9,18)$. However, it should be noted that, while RvD1 increases lesional efferocytosis and clearance of necroptotic cells, these processes were not evaluated in the study by Arnardottir and Thul et al. $(9,18)$. Moreover, SPMs increase fibrous cap thickness in advanced murine lesions, although these changes in plaque morphology were not specifically assessed in the study by Arnardottir and Thul et al. $(9,11)$. Moreover, the hGPR32 $\mathrm{Tg} \times$ Apoe $^{-/-} \times \mathrm{Fpr}^{-/-}$mice were only compared with Apoe ${ }^{-1-} \times \mathrm{Fpr}^{-/-}$ littermates and loss of Fpr2 alone is known to affect atherosclerotic lesion area and stability $(8,15)$. Nonetheless, all aforementioned studies demonstrate atheroprotective roles for SPMs and their receptors and these effects occur independently of changes in cholesterol, indicating that activating proresolving receptors may offer an approach for reducing residual risk in patients already on lipid-lowering therapy.

\section{Regulation of proresolving immune cell subsets by GPR32} RvD1 engages GPR32 to promote macrophage efferocytosis and phagocytosis, 
key functions in resolution (3). Moreover, the RvD1/GPR32 axis modulates leukocyte-EC interactions in response to proinflammatory stimuli $(5,19)$. To obtain additional evidence for a proresolving role of GPR32 in vivo, Arnardottir and Thul et al. utilized a well-characterized model of self-limited peritoneal inflammation. Here, overexpression of hGPR32 in Apoe ${ }^{-} \times \mathrm{Fpr}^{-/-}$mice reduced PMN infiltration in response to zymosan as compared with that in control mice, although the impact of hGPR32 on inflammation resolution in the absence of Apoe deficiency-induced hypercholesterolemia was not specifically evaluated. Notably, AT-RvD1 administration prior to zymosan similarly reduced PMN levels and AT-RvD1 had no effect in Apoe ${ }^{-} \times A l x / F p r 2^{-/-}$mice, demonstrating receptor specificity. Overexpression of hGPR32 markedly increased the macrophage/PMN ratio and increased efferocytosis in vivo, providing definitive evidence that GPR32 is a proresolving receptor. These results are congruent with overexpression of $\mathrm{h} A L X$ / $F P R 2$ in mice, which increases resolution in response to RvD1 (4). Interestingly, hGPR32 Tg mice had higher levels of Alox15, a resolution macrophage marker encoding an enzyme that promotes SPM biosynthesis, as well as lower levels of proinflammatory cytokines Il1 $\beta$ and Tnf $\alpha$. These results are consistent with GPR32-dependent roles of RvD1 in regulating cytokine production in human macrophages (20). In macrophages isolated from hGPR32 Tg mice, AT-RvD1 markedly enhanced phagocytosis of zymosan and bacteria while blunting the uptake of atherogenic oxidized LDL. Functional coupling of the hGPR32 to downstream signaling was further evidenced by phosphorylation of ERK1/2 in response to AT-RvD1 in these macrophages. Although the role of the RvD1/GPR32 axis on T cell subsets was not investigated, the authors note that GPR32 was expressed on T cells in human atherosclerotic lesions and correlated with FOXP3, a Treg marker. Importantly, RvD1 prevents naive $\mathrm{CD}^{+} \mathrm{T}$ cell differentiation into Th1 and Th17 cells and enhances de novo generation and function of $\mathrm{FOXP}^{+}$ Tregs in a GPR32-dependent manner (21). Given that regression of atherosclerosis requires Tregs to modulate macrophage phenotype and efferocytosis, it will be important to further define the cell-specific roles of GPR32 in future studies (22).

\section{Summary and future directions}

Arnardottir and Thul et al. provide evidence of the proresolution and atheroprotective actions of GPR32. Targeting GPR32 with a stable analog, such as benzo-diacetylenic-17R-RvD1-methyl ester, could also be considered, as it is more resistant to metabolic inactivation than native RvD1, yet has similar biological functions (23). Moreover, a recent chemical library screen identified additional GPR32 small molecule chemotype agonists that may offer advantages in terms of stability and scalability (24).

Building on decades of animal studies that show a causal role for inflammation in the progression of atherosclerosis, recent clinical trials demonstrate a reduction in cardiovascular events in patients treated with inhibitors of inflammation (25). Nonetheless, traditional antiinflammatory approaches may impair host defense. Unlike antagonists, SPMs serve as agonists to actively dampen inflammation, promote apoptotic and necrotic cell clearance, engage tissue repair programs, and enhance immune cell-mediated containment of pathogens (1). Thus, proresolving therapeutics have the potential to dampen inflammation and stabilize lesions without compromising host defense. In pursuit of that goal, Arnardottir and Thul et al. provide evidence that GPR32 can potentially serve as a therapeutic target for resolving chronic atherosclerotic inflammation (7).

\section{Acknowledgments}

MS's laboratory is supported in part by NIH grants HL106173, DK122808, DK124782, and GM095467.

Address correspondence to: Matthew Spite, Hale Building for Transformative Medicine, 60 Fenwood Rd., Room 3016K, Boston, Massachusetts 02115, USA. Phone: 617.525.5133; Email: mspite@bwh. harvard.edu.

1. Serhan CN. Pro-resolving lipid mediators are leads for resolution physiology. Nature. 2014;510(7503):92-101.

2. Chiang N, Serhan CN. Structural elucidation and physiologic functions of specialized pro-resolving mediators and their receptors. Mol Aspects Med. 2017;58:114-129.

3. Krishnamoorthy S, et al. Resolvin D1 binds human phagocytes with evidence for prore- solving receptors. Proc Natl Acad Sci U S A. 2010;107(4):1660-1665.

4. Krishnamoorthy S, et al. Resolvin D1 receptor stereoselectivity and regulation of inflammation and proresolving microRNAs. Am J Pathol. 2012;180(5):2018-2027.

5. Norling LV, et al. Resolvin D1 limits polymorphonuclear leukocyte recruitment to inflammatory loci: receptor-dependent actions. Arterioscler Thromb Vasc Biol. 2012;32(8):1970-1978.

6. Sansbury BE, et al. Myeloid ALX/FPR2 regulates vascularization following tissue injury. Proc Natl Acad Sci U S A. 2020;117(25):14354-14364.

7. Arnardottir H, et al. The resolvin D1 receptor GPR32 transduces inflammation resolution and atheroprotection. JClin Invest. 2021;131(24):e142883.

8. Back M, et al. Inflammation and its resolution in atherosclerosis: mediators and therapeutic opportunities. Nat Rev Cardiol. 2019;16(7):389-406.

9. Fredman G, et al. An imbalance between specialized pro-resolving lipid mediators and pro-inflammatory leukotrienes promotes instability of atherosclerotic plaques. Nat Commun. 2016;7:12859.

10. Thul S, et al. Low salivary resolvin D1 to leukotriene $\mathrm{B}_{4}$ ratio predicts carotid intima media thickness: A novel biomarker of non-resolving vascular inflammation. Eur J Prev Cardiol. 2017;24(9):903-906.

11. Viola JR, et al. Resolving lipid mediators maresin 1 and resolvin D2 prevent atheroprogression in mice. Circ Res. 2016;119(9):1030-1038.

12. Welty FK, et al. Regression of human coronary artery plaque is associated with a high ratio of (18-hydroxy-eicosapentaenoic acid + resolvin E1) to leukotriene $\mathrm{B}_{4}$. FASEB J. 2021;35(4):e21448.

13. Merched AJ, et al. Atherosclerosis: evidence for impairment of resolution of vascular inflammation governed by specific lipid mediators. FASEB J. 2008;22(10):3595-3606.

14. Conte MS, et al. Pro-resolving lipid mediators in vascular disease. J Clin Invest. 2018;128(9):3727-3735.

15. Petri MH, et al. The role of the FPR2/ ALX receptor in atherosclerosis development and plaque stability. Cardiovasc Res. 2015;105(1):65-74.

16. Hasturk H, et al. Resolvin E1 (RvE1) attenuates atherosclerotic plaque formation in diet and inflammation-induced atherogenesis. Arterioscler Thromb Vasc Biol. 2015;35(5):1123-1133.

17. Laguna-Fernandez A, et al. ERV1/ChemR23 signaling protects against atherosclerosis by modifying oxidized low-density lipoprotein uptake and phagocytosis in macrophages. Circulation. 2018;138(16):1693-1705.

18. Gerlach BD, et al. Resolvin D1 promotes the targeting and clearance of necroptotic cells. Cell Death Differ. 2020;27(2):525-539.

19. Chatterjee A, et al. Biosynthesis of proresolving lipid mediators by vascular cells and tissues. FASEB J. 2017;31(8):3393-3402.

20. Schmid M, et al. Resolvin D1 polarizes primary human macrophages toward a proresolu- 
tion phenotype through GPR32. J Immunol. 2016;196(8):3429-3437.

21. Chiurchiu V, et al. Proresolving lipid mediators resolvin D1, resolvin D2, and maresin 1 are critical in modulating T cell responses. Sci Transl Med. 2016;8(353):353ra111.

22. Sharma M, et al. Regulatory T cells license macro- phage pro-resolving functions during atherosclerosis regression. Circ Res. 2020;127(3):335-353.

23. Orr SK, et al. Proresolving actions of a new resolvin D1 analog mimetic qualifies as an immunoresolvent. Am J Physiol Lung Cell Mol Physiol. 2015;308(9):L904-L911.

24. Chiang $\mathrm{N}$, et al. Identification of chemotype agonists for human resolvin D1 receptor DRV1 with pro-resolving functions. Cell Chem Biol. 2019;26(2):244-254.

25. Ridker PM. Targeting interleukin-1 and interleukin-6: the time has come to aggressively address residual inflammatory risk. J Am Coll Cardiol. 2020;76(15):1774-1776 